\title{
Does the Animal Origin Influence the Calcification of Xenograft Tissue Heart Valve Substitutes? Comparison between Bovine and Camel Pericardium in a Subcutaneous Rat Model
}

\author{
Fatima Al Harmoodi', Salah Al Shafy ${ }^{2}$, Michel Guichardant ${ }^{3}$, Jean-Jacques Lebrun ${ }^{4}$, \\ Francoise Thivolet ${ }^{5}$, Olivier Jegaden ${ }^{6,7,8^{*}}$ \\ ${ }^{1}$ Department of biomedical engineering, Khalifa University, Abu Dhabi, UAE \\ ${ }^{2}$ Department of cardiac surgery, Zayed Military Hospital, Abu Dhabi, UAE \\ ${ }^{3}$ Chemistry-Biology Health Area, Institut Multidisciplinaire de Biochimie des lipides, Institut National des \\ Sciences Appliquées, Lyon, France \\ ${ }^{4}$ Institute of Analytic Sciences, Centre National de la Recherche Scientifique, Villeurbanne, France \\ ${ }^{5}$ Department of Biology, Hospices Civils de Lyon, Claude Bernard University Lyon1, Lyon, France \\ ${ }^{6}$ Department of Cardiac Surgery, Cleveland Clinic Abu Dhabi, Abu Dhabi, UAE \\ ${ }^{7}$ Claude Bernard University Lyon1, Lyon, France \\ ${ }^{8}$ Cleveland Clinic Lerner College of Medicine-Case Western Reserve University, Cleveland, Ohio, USA \\ Email: ${ }^{*}$ jegadeo@clevelandclinicabudhabi.ae
}

Received 24 August 2015; accepted 21 September 2015; published 24 September 2015

Copyright (C) 2015 by authors and Scientific Research Publishing Inc.

This work is licensed under the Creative Commons Attribution International License (CC BY).

http://creativecommons.org/licenses/by/4.0/

c) (i) Open Access

\begin{abstract}
Objective: To validate the hypothesis that camel pericardium could be more protected than bovine pericardium against calcification process according to the huge difference in their respective lifestyle and lifetime. Methods: Glutaraldehyde (GA) fixed bovine and camel pericardium samples (BP and $\mathrm{CP}$ respectively) were both implanted in $30 \mathrm{New}$ Zealand white rats (2 BP and 2 CP matched specimens in each animal) and explanted after 60 days. Unimplanted GA-fixed samples of both species served as control. Matched implanted samples and unimplanted samples were randomly submitted to elemental analysis by spectroscopy, phospholipid extraction, macroscopic and X-ray examination and histology. Results: At 60 days, calcium and phosphorus content were respectively $9.54 \% \pm 3.1 \%$ and $4.79 \% \pm 1.4 \%$ of tissue dry weight in $B P$, and $12.52 \% \pm 2.7 \%$ and $6.14 \% \pm 1.3 \%$ of tissue dry weight in CP (ns). In X-ray analysis, the calcification score was $1.28 \pm 0.45$ and $2.14 \pm$

"Corresponding author.
\end{abstract}

How to cite this paper: Al Harmoodi, F., et al. (2015) Does the Animal Origin Influence the Calcification of Xenograft Tissue Heart Valve Substitutes? Comparison between Bovine and Camel Pericardium in a Subcutaneous Rat Model. International Journal of Clinical Medicine, 6, 700-709. http://dx.doi.org/10.4236/ijcm.2015.69093 
0.98 in BP and CP samples respectively without significant difference $(\mathrm{p}<0.08)$. In histology, calcifications were lower in BP than in CP: $1.37 \pm 0.85$ vs $2.28 \pm 0.83$ (ns); collagen fibers were better conserved in BP than in CP: $2.4 \pm 0.48$ vs $1.87 \pm 0.78$ (ns), and less disoriented: $25 \%$ vs $62 \%$ (ns). In unimplanted samples, there was a higher but not significant rate of extracted lipids in CP: $5.7 \pm$ 1.8 vs $9.5 \pm 3.8$ nanomoles in PS fraction and $11.3 \pm 3.7$ vs $19 \pm 7.7$ nanomoles in total fatty acids, in $\mathrm{BP}$ and CP samples respectively. All results were in conjunction and demonstrated a higher but not significant rate of mineralization in camel pericardium after implantation, which could be related to a higher but not significant basic rate of phospholipid and fatty acids. Conclusion: This experiment study in a subcutaneous rat model has failed to valid our hypothesis. Because the differences observed between bovine and camel pericardium did not reach the significance, at the best, there is no difference between both species and at the worst, camel pericardium has a higher rate of the phosphatidylserine fraction of phospholipid, and is more sensitive and prompt to calcification.

\section{Keywords}

\section{Pericardium, Calcification, Bovine, Camel, Xenograft Tissue, Heart Valve Substitutes}

\section{Introduction}

Structural dysfunction, due to calcification and non-calcific deterioration, is the major cause of failure of bioprosthetic heart valves. Glutaraldehyde (GA) was introduced for fixation of bioprosthetic valves to decrease immunogenecity, increase crosslinking of connective tissue proteins, and increase strength and durability [1]. Consequently, it has been used extensively for the preservation of bovine pericardium derived valves and porcine aortic valves. However, it has been found that GA fixation stimulates dystrophic calcification, as a consequence of xenograft cells devitalization and a toxic effect of unstable cross-linking. The mechanism of mineralization of GA-fixed bioprosthesis consists of attraction and precipitation in which activated phosphatases bind to calcium upon lipid-based cell debris, which are full of phospholipids [2] [3]. Despite the controversies around the different effects of GA on the calcification process [4] [5], GA-fixed tissue remains the basic concept of xenograft engineering and GA is the standard fixative with no alternative in view. Nevertheless, additional anticalcificant treatments have been developed to block calcification at the cellular level and to provide better structural integrity [6] [7].

Regardless of the fixation and the post-fixation treatments of the pericardium, we have considered that the animal origin of the xenograft could influence the process of calcification and we have tested this hypothesis in a comparison between bovine and camel origin. The main objective of this study was finding a potential difference in the degree of calcification for GA-fixed bovine (BP) and camel pericardium (CP) in a subcutaneous rat model. The organisms live a diverse lifestyle with a variance of eating habits, habitats and life expectancy which could involve variety of components present in $\mathrm{BP}$ and $\mathrm{CP}$, being able to influence the calcification process of their respective tissue.

\section{Material and Methods}

\subsection{Tissue Preparation}

Samples of bovine pericardium (BP) and camel pericardium (CP) were obtained from a local abattoir. The removal of any adherent adipose tissue was followed by rinsing in $0.9 \%$ cold physiological saline. Samples were pre-fixed with a high concentration of glutaraldehyde (0.625\%) for a period of 30 minutes. To remain consistent, using a round cutter instrument, the samples were assembled into $0.8 \mathrm{~cm}^{2}$ sections. Subsequently, all samples were stored by immersion in $0.625 \% \mathrm{GA}$ at $4^{\circ} \mathrm{C}$ for 24 hours.

\subsection{Subcutaneous Implantation}

The round samples, both BP and CP, were implanted subcutaneously in thirty 2-week-old New Zealand white rats. The surgical procedure was conducted under general anesthesia by the effect of ether. The dorsal area was shaved and disinfected prior to implantation of tissue samples which were rinsed in saline for $3 \mathrm{~min}$ to remove 
any residual storage solution. Each rat received 4 samples, 2 BP and 2 CP; each sample was implanted through an incision of $1 \mathrm{~cm}$ into the dorsal wall. The unimplanted remaining samples, BP and CP, were stored in $0.625 \%$ $\mathrm{GA}$ at $4^{\circ} \mathrm{C}$ as a control group. All animals utilized in this research were cared for according to the policies and principles established by the Animal Welfare Act and the NIHGuide for Care and Use of Laboratory Animals. After 60 days, the animals were sacrificed using an overdose of barbiturates and the samples were retrieved and oriented to three different analysis. For elemental analysis, 10 samples, 5 BP and 5 CP, were stored under cold conditions. For lipid extraction and fatty acid analysis, 4 samples, 2 BP and 2 CP, were frozen using liquid nitrogen. For morphology and radiography, 10 other samples were collected, $5 \mathrm{BP}$ and $5 \mathrm{CP}$, and then they were fixed in $4 \%$ formaldehyde for histologic analysis. The BP and CP samples explanted were matched by animals to avoid any variations due the hosts and were identified numerically. Concurrently, the unimplanted and stored during the same 60 days BP and CP samples were rinsed in saline for $3 \mathrm{~min}$ to remove any residual storage solution, and then were oriented to the different analysis, as the explanted samples; they were identified as 0 (BP0, CP0).

\subsection{Elemental Analysis}

Calcium contents were assessed by atomic absorption spectroscopy. Samples were dried to a content weight in a desiccator oven and hydrolyzed with $\mathrm{HNO}_{3}(0.75 \mathrm{~mol} / \mathrm{l})$ at $68^{\circ} \mathrm{C}$ for 15 hours. After centrifuging at $2500 \times \mathrm{g}$, the fluid was removed, diluted and the calcium content was determined using the atomic absorption spectrometer UNICAM Solaar 989. A calibration curve was plotted using a set of crescent concentrations of a calcium standard solution and distilled water blank. Calcium contents, expressed as percentage of tissue dry weight, represent the mean of at least three different spectrometric determinations. Phosphorous contents, expressed, as percentage of tissue dry weight, were assessed by inductively coupled analysis.

\subsection{Phospholipid Analysis}

The lipids and then the phospholipids were extracted and separated into different classes. The phosphatidyl serine fraction (PS) was transesterified and the fatty acid methyl esters were analyzed by gas chromatography. Each fatty acid is expressed in \% relative to the total fatty acids in the fraction PS.

\subsection{Macroscopic Examination and X-Ray Analysis}

The morphology of each sample was assessed before submission to X-ray analysis using mammography technique. For X-ray analysis the presence of calcium deposits was quantified on the basis of a 0 - 4 score as follows: 0 = absent; 1 = focal, pinpoint, $<1 \mathrm{~mm}$ of diameter; 2 = focal, $>1 \mathrm{~mm}$ of diameter or pinpoint multiple; $3=$ multiple $>1$ mm diameter; 4 = massive deposition.

\subsection{Histology}

The pericardial samples, BP and CP, were fixed in $4 \%$ formaldehyde, processed, and stained with hematoxylineosin (H\&E) and von Kossa staining. The following parameters were evaluated:

- Presence, amount and type of mineralization (nodular and laminar, whether calcium deposits occurred on cells, collagen fibers or both) on Von Kossa stained sections.

- Presence, amount and type of inflammatory reaction (either granulomatous, lymphocytic or neutrophilic) on hematoxylin-eosin stained sections.

A semi quantitative method was applied on histologic sections to evaluate the amount of mineralization and inflammatory infiltrate: (0) absent; (1) minimal; (2) mild; (3) moderate; and (4) severe.

\subsection{Statistical Analysis}

Quantitative data were presented as mean \pm SEM. The statistical analysis included a variance check (ANOVA); The nonparametric Mann-Whitney $U$ and Wilcoxon rank sum tests were used with a pairwise comparison of $95 \%$ confidence intervals. The probability was set at $\mathrm{p}<0.05$ for significance.

\section{Results}

All animals except one survived to the surgical procedure and they could be sacrificed at 60-day follow-up. 
Implanted pericardium samples were matched by animal and then randomly distributed to the different analyses. Unimplanted samples were randomly distributed to the different analyses after storage.

\subsection{Elemental Analysis}

In unimplanted samples, calcium and phosphorus contents were at the limit of detection without difference between bovine and camel origin (Table 1). In implanted samples, calcium and phosphorus contents ranged from $4.22 \%$ to $15.93 \%$ and $2.33 \%$ to $7.70 \%$ respectively. In matched implanted samples, calcium and phosphorus contents were higher in camel pericardium (Table 1) showing a higher mineralization process in this group. However, the difference between BP and CP were not significant (Figure 1).

\subsection{Phospholipid Analysis}

The phospholipid analysis was done on 8 unimplanted samples (4 BP and 4 CP) and 4 matched implanted samples (2 BP and $2 \mathrm{CP}$ ). In unimplanted bovine and camel samples, the phosphatidylserine fraction ranged from 4.6 to 8.5 Nanomoles and from 4.6 to 15.2 Nanomoles respectively, and the total fatty acids in PS fraction, after new calibration, ranged from 6.9 to 16.9 Nanomoles and from 9.2 to 30.4 Nanomoles respectively, showing a higher rate of extracted lipids in camel samples: in mean, $5.7 \pm 1.8$ vs $9.5 \pm 3.8$ (ns) Nanomoles in PS fraction and $11.3 \pm 3.7$ vs $19 \pm 7.7$ (ns) Nanomoles in total fatty acids, in bovine and camel samples respectively. However, the distributions of the fatty acids were comparable between camel and bovine pericardium samples (Figure 2). In implanted samples, results were similar: in mean, $3.5 \pm 1$ vs $16.8 \pm 8.5$ (ns) Nanomoles in PS fraction and $7.1 \pm 2$ vs $33.6 \pm 17$ (ns) Nanomoles in total fatty acids, in bovine and camel samples respectively with a comparable distribution of fatty acids (Figure 2). Interestingly, PS fraction and total fatty acids were in implanted samples lower in BP and higher in CP in comparison with respective unimplanted samples.

Table 1. Calcium and Phosphorous contents in bovine (BP) and camel (CP) pericardium matched samples. 0 indicates unimplanted samples; 1 to 5 indicate implanted samples. Results are expressed in \% of tissue dry weight.

\begin{tabular}{|c|c|c|c|c|c|c|c|c|}
\hline Content & Samples & 0 & 1 & 2 & 3 & 4 & 5 & Mean \\
\hline \multirow{2}{*}{ Calcium } & $\mathrm{BP}$ & $<300$ ppm & 8.59 & 11.21 & 10.12 & 13.54 & 4.22 & $9.54 \pm 3.1$ \\
\hline & $\mathrm{CP}$ & $<300$ ppm & 13.62 & 12.04 & 7.71 & 13.32 & 15.93 & $12.52 \pm 2.7$ \\
\hline \multirow{2}{*}{ Phosphorous } & BP & 0.09 & 4.34 & 5.61 & 5.01 & 6.64 & 2.33 & $4.79 \pm 1.4$ \\
\hline & $\mathrm{CP}$ & 0.10 & 6.66 & 5.93 & 3.81 & 6.49 & 7.70 & $6.14 \pm 1.3$ \\
\hline
\end{tabular}

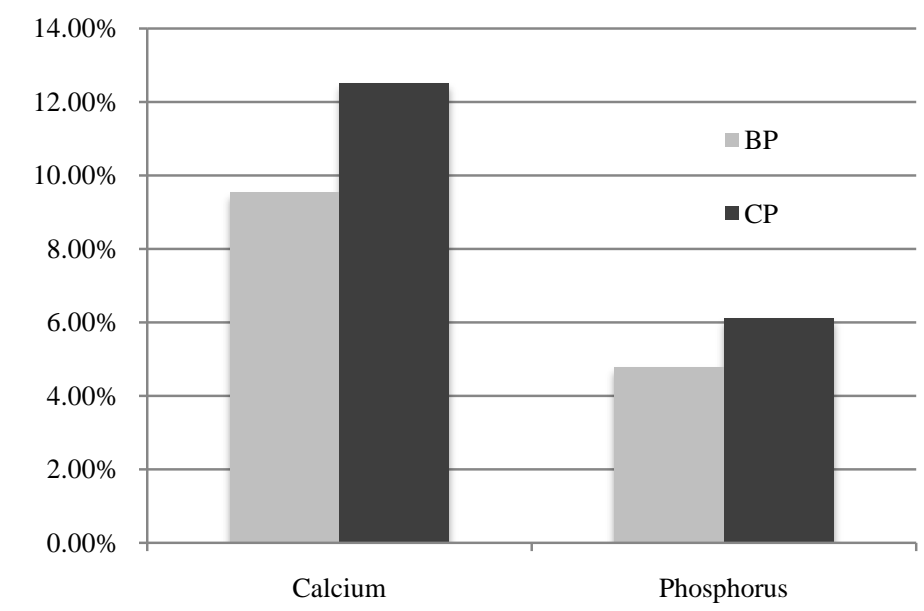

Figure 1. Histogram of calcium and phosphorous content expressed as percentage tissue dry weight. BP, bovine pericardium, CP, camel pericardium. 



Figure 2. Histogram of means for fatty acids present in unimplanted and implanted BP and CP samples. BP, bovine pericardium, CP, camel pericardium.

\subsection{Macroscopic Examination and X-Ray Analysis}

The macroscopic aspect of unimplanted and stored samples looks like fine; all are soft, supple, nongritty and well preserved without obvious difference between bovine and camel samples (Figure 3(a) \& Figure 3(b)). On the contrary, all implanted samples look like small bones, more and less rigid, thickened, hard and gritty, as the consequence of the mineralization and calcification process during the implantation period; there was no visible difference between bovine and camel samples (Figure 3(c) \& Figure 3(d)).

The X-ray analysis involved 14 matched implanted samples (7 BP and 7 CP); the presence of calcium deposit was quantified on the basis of a 0 - 4 score (Figure 4) by two independent observers. The calcification score was $1.28 \pm 0.45$ and $2.14 \pm 0.98$ in BP and CP samples respectively without significant difference $(\mathrm{p}<0.08)$.

\subsection{Histology}

Histological analysis involved 4 unimplanted samples (2 BP and 2 CP). There was no difference in the cell components: all pericardium samples had absence of lymphocytes, neutrophils, giant cells and calcification. The 


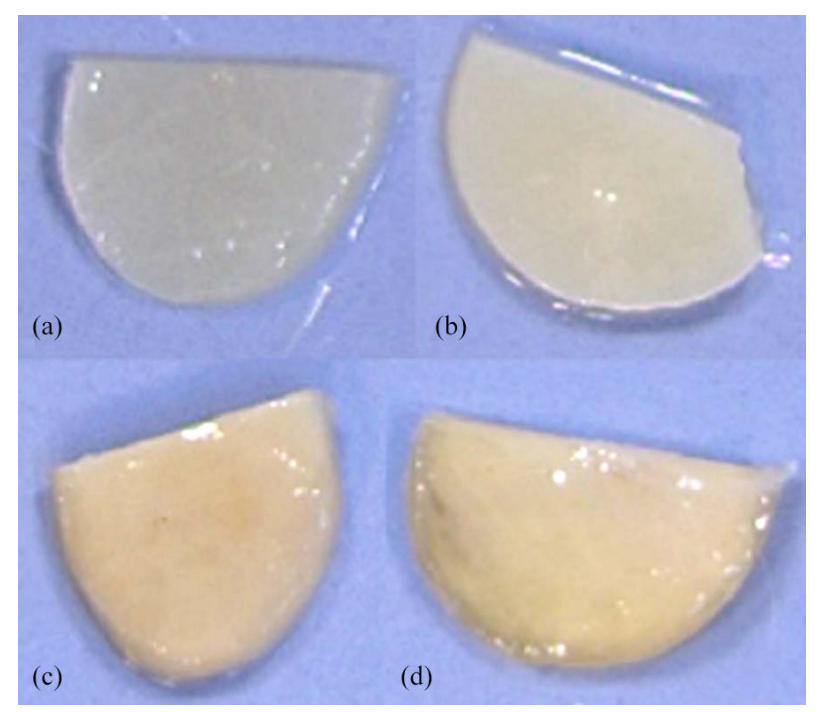

Figure 3. Morphology of pericardium samples before specific analysis. (a) BP unimplanted sample (b) CP unimplanted sample (c) BP implanted sample (d) CP implanted sample. $\mathrm{BP}$, bovine pericardium, $\mathrm{CP}$, camel pericardium.



(a)

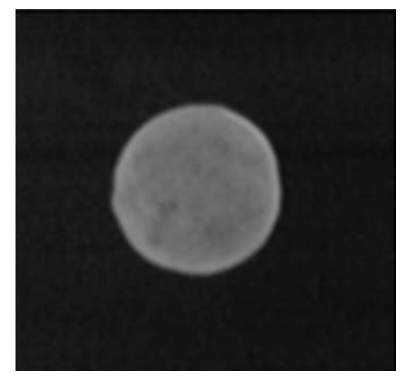

(b)

Figure 4. Mammography X-ray analysis of implanted samples. (a) shows a CP implanted sample with obvious calcium deposits with a score of 4 . (b) shows a CP implanted sample with calcifications score of $1 . \mathrm{BP}$, bovine pericardium, $\mathrm{CP}$, camel pericardium.

collagen fibers were present at a score of 3 and were well oriented in all samples (Figure 5, IV).

Histological analysis involved 16 matched implanted samples (8 BP and 8 CP). The different microscopic aspects observed are illustrated in Figure 5 and semi-quantitative scores are summarized in Figure 6. Calcifications were lower in BP than in CP: $1.37 \pm 0.85$ vs $2.28 \pm 0.83$ (ns); collagen fibers were better conserved in BP than in CP: $2.4 \pm 0.48$ vs $1.87 \pm 0.78$ (ns) and less disoriented: $25 \%$ vs $62 \%$ (ns). The inflammatory infiltration by lymphocytes and giant cells was overall low with a light difference between BP and CP (ns).

\section{Comments}

Calcification is an important factor in clinical dysfunction of bioprosthetic heart valves. The onset of calcification has been postulated to originate from an electrostatic attraction between the acid phospholipids of the connective tissue and calcium. Accelerated calcification is associated with young recipient age, glutaraldehyde (GA) fixation, and high mechanical stress. Xenograft heart valves treated with GA and mounted in a rigid stent are the most widely used cardiac bioprostheses. Although stable cross-links in cellular and extracellular matrix proteins are considered to reduce immunogenicity, GA treatment has been demonstrated to increase calcification by fixing cellular debris in place [1] [2]. 


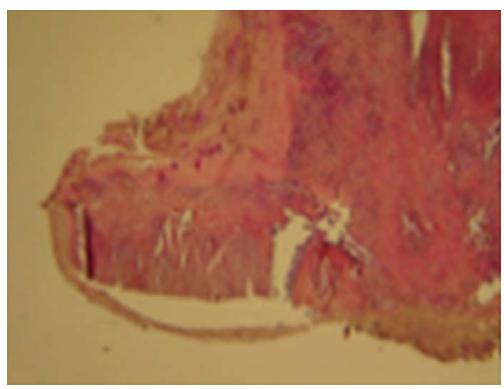

(a)



(a)

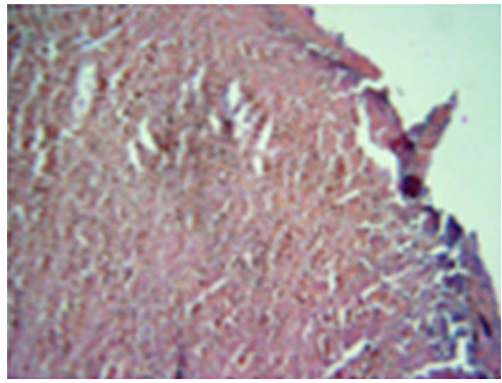

(a)

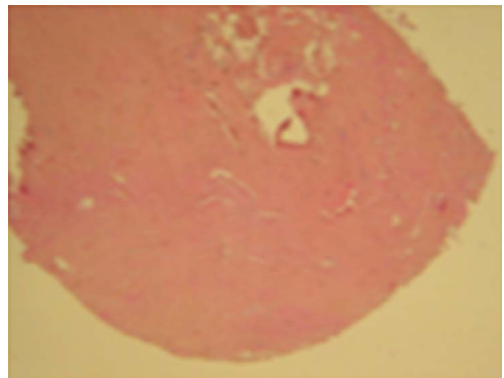

(a)

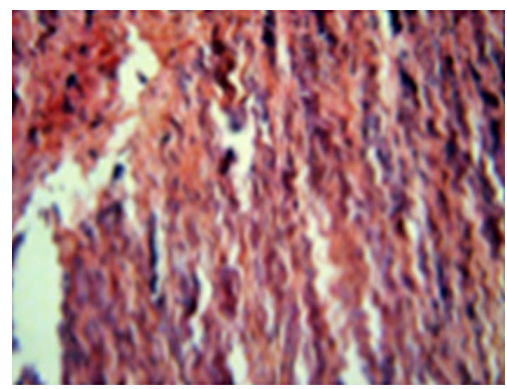

(b)

(1)

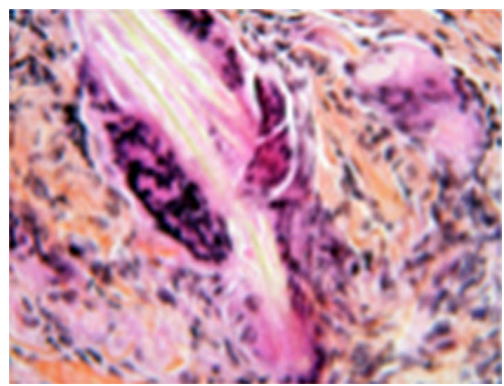

(b)

(2)

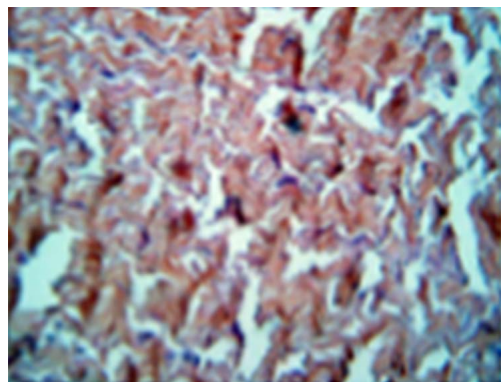

(b)

(3)



(b)

(4)

Figure 5. Histology of pericardium samples. (1) Implanted camel pericardium (a) Light microscopy in stained with H\&E showing mild disruption of collagen fibers of the pericardial matrix; (b) High microscopy with Von Kossa staining showing severe calcifications (brown deposits). (2) Implanted bovine pericardium stained with H\&E, (a) Light microscopy showing the presence of inflammatory cells (lymphocytes and giant cells); collagen fibers of the pericardial matrix remaining well oriented; (b) High microscopy showing giant cells. (3) Implanted camel pericardium with Von Kossa staining (a) Light microscopy and (b) High microscopy showing severe disruption of collagen fiber of the pericardial matrix with severe calcification (brown deposits). (4) Unimplanted camel pericardium (a) stained with H\&E showing a normal pericardial matrix with well-oriented collagen fibers and without inflammatory cell; (b) Von Kossa staining showing no calcification. 




Figure 6. Histogram of mean scoring for microscopic examinations of implanted samples. BP, bovine pericardium, CP, camel pericardium.

Cells are the predominant location of mineralization and the usual pretreatment of commercially available bioprostheses with GA, done to improve tissue durability, also potentiate calcification [4] [8]. GA fixation devitalizes but does not remove connective tissue cells that are prone to be the initial site of calcium deposition. Calcification of the extracellular matrix structural proteins collagen and elastin has been studied; collagen and elastic fibers can serve as nucleation sites for calcium phosphate minerals, independent of cellular components [9].

Tremendous efforts have been made to solve this problem and numerous experiments have been carried out to investigate different calcium mitigating agents. Nowadays, all new anticalcificant treatments aim at washing residual GA out; thereafter, either additional surfactants are applied or methods to block calcification at the cellular level or to extract cells are used [6] [10]. At present, not all details of the different treatments are fully disclosed.

Significant correlations between phospholipid levels and calcification have been well established and phospholipids appear to be a significant contributor to the calcification of bioprosthetic tissue [2] [3]. Other mechanisms may also contribute to the observed calcification, as residual aldehyde toxicity and mechanical and cellular factors. Different lipoprotein profiles, potential differences in calcium metabolism, hematologic differences may play a role in the onset of calcification [11] [12].

The animal origin of the xenograft could also influence the process of calcification [13]. We have tested this hypothesis in a comparison between bovine and camel origin. The organisms live a diverse lifestyle with a variance of eating habits, habitats and life expectancy which could involve variety of components present in BP and CP, able to influence the calcification process of their respective tissue. The difference in lifestyle between the two species is the huge, that a significant difference in the calcification process of pericardium could be expected. This study used the subcutaneous implantation of specimens. The subcutaneous model has emerged as a technically convenient and economically advantageous vehicle for investigating host and implant determinants and mechanisms of mineralization, as well as for screening potential strategies for inhibition of calcification [11] [13] [14]. The rat subcutaneous implant is a favorable low cost model with easy availability.

In our experiments, a difference in rate of calcification of the implanted specimens between bovine and camel pericardium origin was observed. The calcium measurements using spectroscopy analysis were in conjunction with the X-ray analysis and the histological score of calcification of samples: all results demonstrated a higher rate of mineralization in camel pericardium specimens. The phospholipid analysis was focused on phospatidylserine fraction and corresponding fatty acids in unimplanted samples, showing higher levels in camel pericardium, with a similar distribution of fatty acids. All differences observed between bovine and camel pericardium did not reached the level of significance, probably as a consequence of the limited number of specimens analyzed. However, even if the results observed were not in the expected direction, this study has confirmed that donor species could influence calcification of tissue valve substitutes [13] and correlation between phospholipid levels and mineralization [3]. Only Phospatidylserine fraction was analysed because it has been described as the 
main phospholipid involved in the process of calcification [1] and also to limit the cost of the study. Interestingly, the distributions of fatty acids were similar in implanted and unimplanted samples, and in bovine and camel origin, showing that volume of phospholipid is maybe more determinant than specificity in the process of calcification. However, it is well established that additional treatment to remove phospholids from the xenograft tissue or to neutralizes the toxic residual aldhehyde groups have failed to prevent mineralization; the role of residual matrix immunoreactivity could be predominant.

Tissue engineering heart valves constructed from a xenogeneic or an allogeneic heart valve scaffold eventually covered with autologous endothelial cells are considered to overcome all these obstacles [10] [15]. Several studies have led to the selection of human allograft, not xenografts, as a basis for decellularization technologies designed to obtain functioning valve extracellular matrix (ECM) scaffolds for tissue engineering of heart valves, a goal which involves a unique combination of biological, engineering, and technological hurdles [12] [15]. The tissue engineering heart valve is the prototype multidisciplinary collaborative project that is virtually ideal as the archetype for the new era of bioengineered solutions to complex cardiac diseases.

A limitation of the study is a lack of investigation on mechanical properties like elasticity and strength as well as resistance to enzymatic and cellular digestion. Such investigations would have been planned if this study had been able to demonstrate a lower mineralization process in camel pericardium and so a possible benefit to develop its use.

\section{Conclusion}

Calcification and deterioration of GA-fixed heart valve substitutes remain a concern. We made the hypothesis that camel pericardium could be more protected than bovine pericardium against calcification according to the huge difference in their respective lifestyle and lifetime. Our experiment study in a subcutaneous rat model has failed to valid this hypothesis. Because the differences observed between bovine and camel pericardium did not reach the significance, at the best, there is no difference between both species and at the worst, camel pericardium is more sensitive and prompt to calcification, with a higher rate of the phosphatidylserine fraction of phospholipid. These observations led us to interrupt our research in camel tissue substitute and to orient them to tissue engineering heart valves which could be more promising according to the lower impact of immunological and enzymatic reactions.

\section{References}

[1] Schoen, F.J. and Levy, R.J. (2005) Calcification of Tissue Heart Valve Subsitutes: Progress toward Understanding and Prevention. The Annals of Thoracic Surgery, 79, 1072-1080. http://dx.doi.org/10.1016/j.athoracsur.2004.06.033

[2] Levy, R.J. (1994) Glutaraldehyde and the Calcification Mechanism of Bioprosthetic Heart Valves. The Journal of Heart Valve Disease, 3, 101-104.

[3] Cunanam, C.M., Cabiling, C.M., Dinh, T.T., Shen, S.S., Tran-Hata, P., Rutledge, J.H. and Fishbein, M.C. (2001) Tissue Characterization and Calcification Potential of Commercial Bioprosthetic Heart Valves. The Annals of Thoracic Surgery, 71, S417-S421. http://dx.doi.org/10.1016/S0003-4975(01)02493-6

[4] Gong, G., Ling, Z., Seifter, E., Factor, S.M. and Frater, R.W.M. (1991) Aldehyde Tanning: The Villain in Bioprosthetic Valve Calcification. European Journal Cardio-Thoracic Surgery, 5, 288-293. http://dx.doi.org/10.1016/1010-7940(91)90037-K

[5] Vincentelli, A., Latremouille, C., Zegdi, R., Shen, M., Lajos, P.S., Chachques, J.C. and Fabiani, J.N. (1998) Does Glutaraldehyde Induce Calcification of Bioprosthetic Tissues? The Annals of Thoracic Surgery, 66, S255-S258. http://dx.doi.org/10.1016/S0003-4975(98)01098-4

[6] Walter, T., Falk, V., Autschbach, R., Diegeler, A., Rauch, T., Weigl, C., Gunther, B., van Son, J.A. and Mohr, F.W. (1998) Comparison of Different Anticalcification Treatments for Stentless Bioprostheses. The Annals of Thoracic Surgery, 66, S249-S254. http://dx.doi.org/10.1016/S0003-4975(98)00985-0

[7] Carpentier, S.M., Chen, L., Shen, M., Fornes, P., Martinet, B., Quintero, L.J., Witzel, T.H. and Carpentier, A.F. (1998) Heat Treatment Mitigates Calcification of Valvular Bioprostheses. The Annals of Thoracic Surgery, 66, S264-S266. http://dx.doi.org/10.1016/S0003-4975(98)01101-1

[8] Hoffmann, D., Gong, G., Liao, K., Macaluso, F., Nikolic, S.D. and Frater, R.W.M. (1992) Spontaneous Host Endothelial Growth on Bioprostheses. Influence of Fixation. Circulation, 86, II75-II79.

[9] Hopkins, R.A. (2005) Tissue Engineering of Heart Valves. Decellularized Valve Scaffolds. Circulation, 111, 27122714. http://dx.doi.org/10.1161/CIRCULATIONAHA.104.527820 
[10] Schoen, F.J., Levy, R.J., Hilbert, S.L. and Bianco, R.W. (1992) Antimineralization Treatments for Bioprosthetic Heart Valves. The Journal of Thoracic and Cardiovascular Surgery, 104, 1285-1288.

[11] Neethling, W.M., Glancy, R. and Hodge, A. (2010) Mitigation of Calcification and Cytotoxicity of a Glutaraldehyde Preserved Bovine Pericardial Matrix: Improved Biocompatibility after Extended Implantation in the Subcutaneous Rat Model. The Journal of Heart Valve Disease, 19, 778-785.

[12] Rieder, E., Seebacher, G., Kasimir, M.T., Eichmair, E., Winter, B., Dekan, B., Wolner, E., Simon, P. and Weigel, G. (2005) Tissue Engineering of Heart Valves. Decellularized Porcine and Human Valve Scallops Differ Importantly in Residual Potential to Attract Monocytic Cells. Circulation, 111, 2792-2797. http://dx.doi.org/10.1161/CIRCULATIONAHA.104.473629

[13] Carpentier, S.M., Monier, M.H., Shen, M. and Carpentier, A.F. (1995) Do Donor or Recipient Species Influence Calcification of Bioprosthetic Tissues? The Annals of Thoracic Surgery, 60, S328-S331. http://dx.doi.org/10.1016/0003-4975(95)00244-F

[14] Pettenazzo, E., Valente, M. and Thiene, G. (2008) Octanediol Treatment of Glutaraldhehyde Fixed Bovine Pericardium: Evidence of Anticalcification Efficacy in the Subcutaneous Rat Model. European Journal Cardio-Thoracic Surgery, 34, 418-422. http://dx.doi.org/10.1016/j.ejcts.2008.05.012

[15] Mendelson, K. and Schoen, F.J. (2006) Heart Valve Tissue Engineering: Concepts, Approaches, Progress, and Challenges. Annals of Biomedical Engineering, 34, 1799-1819. http://dx.doi.org/10.1007/s10439-006-9163-z 